\title{
WIND-FIELD AND POLLUTION-DISPERSION SIMULATION IN A STREET CANYON IN HELSINKI WITH ADREA-HF CODE
}

\author{
P. NEOFYTOU* \\ A. VENETSANOS \\ J.G. BARTZIS
}

Received: $15 / 12 / 05$

Accepted: 15/05/06

\author{
Environmental Research Lab. , INT-RP \\ NCSR Demokritos \\ Aghia Paraskevi, 15310 Athens, Greece
}

*to whom all correspondence should be addressed: e-mail: panosn@ipta.demokritos.gr

\begin{abstract}
ADREA-HF, which is a Computational Fluid Dynamics (CFD) code, is utilised in order to numerically study the flow and concentration fields within a street-canyon area. The selected site is Runeberg Str., a typical urban street canyon with an aspect ratio of approximately 1:1 in Helsinki, Finland. The ADREA-HF model is a transient, non-hydrostatic, dense transport code, especially developed for dispersion modelling of buoyant or passive gases over complex terrain in local scale. It solves the 3D unsteady Reynolds Averaged Navier-Stokes (RANS) equations treating complex multi-building domains with a porosity formulation. For modelling turbulence a one-equation eddy-viscosity model is used. The numerical results illustrate the flow and concentration fields within the canyon and also show the influence of the detailed geometry, such as, that of the street junction situated at the northern end of the canyon, and that of the boulevard at the southern end.
\end{abstract}

KEYWORDS: Simulation, Street Canyon, Computational Fluid Dynamics

\section{INTRODUCTION}

The role of Computational Fluid Dynamics (CFD) as a tool for assessing the pollutiondispersion impact on the environment within inhabited areas is increasing due to cost effectiveness combined with accuracy of predictions. Furthermore the limit of pollutant levels set by the World Health Organisation has led to an increased research activity as to the specification of the influence of car emissions on the air quality in urban street canyons. Urban street canyons consist of building complexes on either side of the street and induce flow recirculations and/or stagnant conditions thus prohibiting the dispersion of pollutants away from inhabited areas.

With respect to CFD applications on environmental flows, a review was carried out by Vardoulakis et al., $2003^{6}$ that includes evaluation of several CFD methods applied in meteorological, wind-tunnel and street-canyon studies. In addition, Walton et al.,2002 pursued LES (Large-Eddy Simulation) for the problem of mean flow and turbulence in cubic street canyons. Their results show good agreement between simulations and experimental data. Finally, CFD computations using the ADREA-HF code have been carried out by Neofytou et al., $2004^{4}$ in order to parametrically study the pollution in a street canyon by assuming different wind directions.

Model validation by comparing with measurements was carried out by Kukkonen et al., 2001; $2003^{2,3}$ in a street canyon (Runeberg Street) in Helsinki. Concentrations of $\mathrm{CO}_{2}, \mathrm{NO}_{\mathrm{x}}$ and $\mathrm{O}_{3}$ were provided from a measurement campaign in 1997 and were compared with predictions by the OSPM model, which qualitatively reproduced the observed behaviour in a consistent manner.

The current study is carried out in the framework of the Optimised Expert System for Conducting Environmental Assessment of Urban Road Traffic (OSCAR) project. This project aims at assessing the environmental impact of road traffic in terms of traffic flows, emissions and air pollution. The data with respect to source of emissions and ambient wind speed and 
direction were obtained from campaigns carried out in Runeberg Street in Helsinki, Finland in 2003-2004. The numerical predictions are carried out using the CFD code ADREA-HF ${ }^{1}$, which also has been used in the past for environmental flow predictions ${ }^{4,5,7}$.

\section{METHODOLOGY}

The methodology consisted in solving the transient, Reynolds averaged, mass and momentum 3D conservation equations for the mean flow and the mass fraction conservation equation for the pollutant dispersion, until steady state conditions were reached. Boundary conditions for the problem were zero gradient and given value for the inflow boundaries, zero gradient for the outflow boundaries, wall functions for velocities at the buildings' surfaces and ground and finally zero vertical velocity at the top of domain.

The computational domain that includes all buildings in the area surrounding Runeberg Street is constructed using actual coordinates provided by the Helsinki Metropolitan Area Council

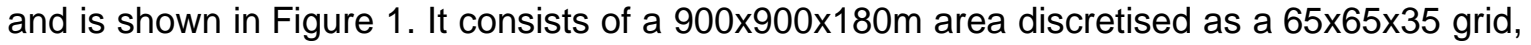
which is refined near the street-canyon location in order to more accurately capture the wind field and concentration distribution.

The monitoring campaign in Runeberg Street was conducted in 2003 - 2004 (19.2.2003 31.12.2004). The aspect ratio of the street canyon is approximately $1: 1$ and the average weekday traffic volume is approximately 23000 vehicles/day at Runeberg Street. Winddirection and wind-speed data are available with the time resolution of one minute. The traffic volumes for each link in the studied area were obtained from a traffic demand modelling system developed by the Helsinki Metropolitan Area Council ${ }^{9}$.

Three different datasets were selected, each covering a 15 minute time period. Each dataset provides values for wind speed and direction at roof level. In addition, traffic emissions are provided for every street at a resolution of one hour. Hence, there was one value of emission for every street representing the selected time period of the dataset. The parameters from each dataset do not substantially fluctuate within the 15-minute period and therefore a mean value was derived for each parameter. The aforementioned values for wind speed and direction were used as input data for calculations in order to have realistic data with respect to the estimation of ambient wind attributes. In addition, the source of pollution from the streets in the vicinity of Runeberg street (Fig. 2) was modelled for each dataset as an area source along the street emitting homogenously and with constant rate. Therefore three different cases, each corresponding to a dataset, were studied. These are shown in Table 1 with the emissions from each traffic link expressed in $\mathrm{g} \mathrm{km}^{-1} \mathrm{~h}^{-1}$.

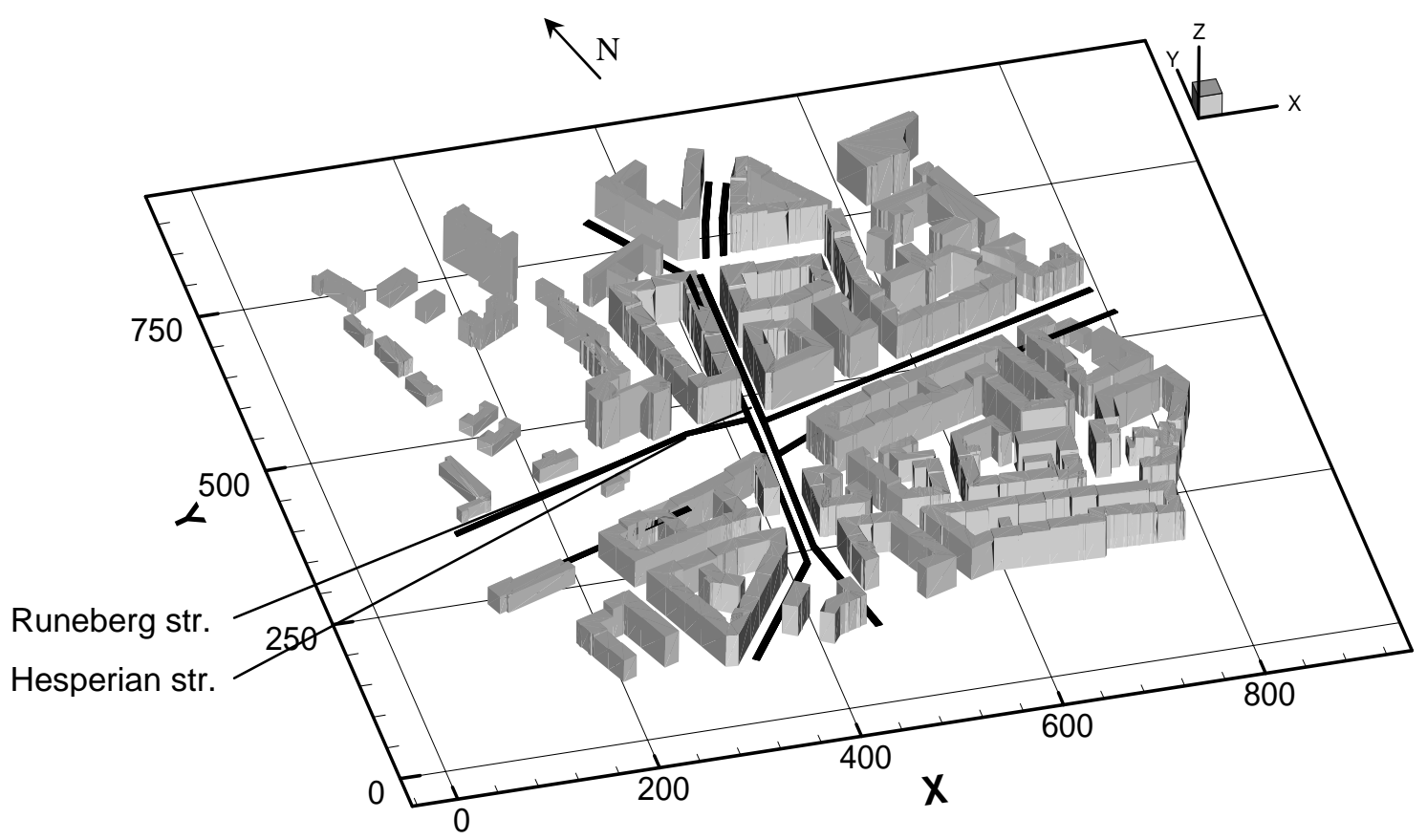

Figure 1. Computational domain of the Runeberg str. area 
Table 1. Overview of datasets with the traffic-link (TL) emissions (Fig. 2) in $\mathrm{g} \mathrm{km}^{-1} \mathrm{~h}^{-1}$.

\begin{tabular}{ccccccccccc}
\hline Dataset & $\begin{array}{c}\text { WS } \\
\left(\mathrm{m} \mathrm{s}^{-1}\right)\end{array}$ & $\begin{array}{c}\text { WD } \\
(\mathrm{deg})\end{array}$ & TL1 & TL2 & TL3 & TL4 & TL5 & TL6 & TL7 & TL8 \\
\hline 1 & 1.69 & 270.3 & 408.9 & 53.0 & 1424.6 & 1779.4 & 25.8 & 1729.3 & 639.7 & 598.5 \\
\hline 2 & 2.40 & 254.4 & 408.9 & 53.0 & 1424.6 & 1779.4 & 25.8 & 1729.3 & 639.7 & 598.5 \\
\hline 3 & 2.14 & 260.6 & 666.6 & 84.0 & 1882.7 & 2493.8 & 35.0 & 2420.0 & 1019.6 & 794.4 \\
\hline
\end{tabular}

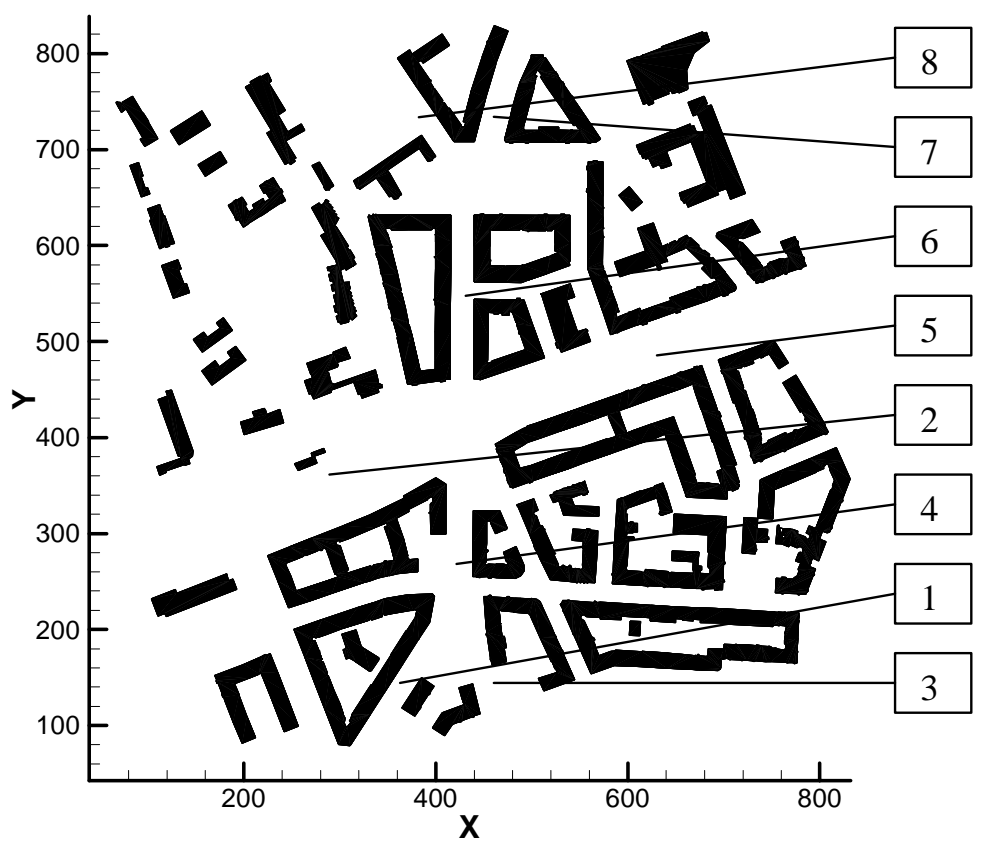

Figure 2. Overview of computational domain with numbered traffic links

\section{RESULTS AND DISCUSSION}

First, it is very interesting to see how the concentration distribution is behaving with respect to the flow field. The comparison is carried out between datasets 1 and 2, for which the same source of emissions is assumed. The relative concentration distribution is shown in Figure 3 for the height of $z=4 \mathrm{~m}$. First, it can be seen that Hesperian Street barely plays any role to the pollution in its vicinity and that can be attributed to both the fact that Hesperian Street is an open area so the pollutants are more easily convected and diffused away and to the fact that it has less traffic compared to Runeberg Street. Thus, the pollution from the latter is more prominent.
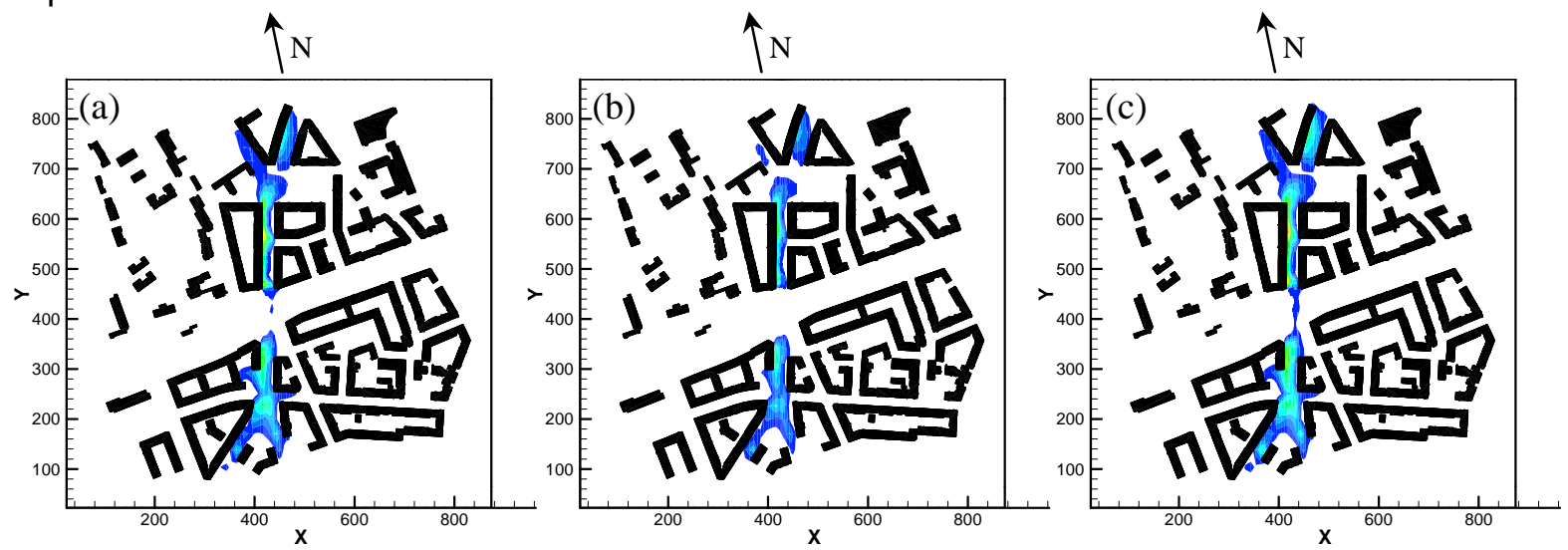

Figure 3. Concentration distribution for NOx at $z=4 m$ for cases: (a) 1; (b) 2; (c) 3 
Furthermore, it can be seen that the concentration levels are relatively higher for case 1 compared to case 2 at $z=4 \mathrm{~m}$. This can be explained from the wind field for that height (Fig. 4) where the wind speeds within the street canyon are relatively higher for case 2 and therefore the dispersion mechanism is more intense. This is caused not only from the higher freestream velocity for case 2 that causes more marked street-canyon effects but also from the fact that the wind direction, which is for this case more aligned with Hesperian Street allows wind to enter from Hesperian Street into Runeberg Street thus increasing further the velocity within the latter. Case 3 exhibits the relatively highest concentration levels among all cases due to the higher emissions assumed although the wind velocity within the canyon is between the levels of the other two cases.
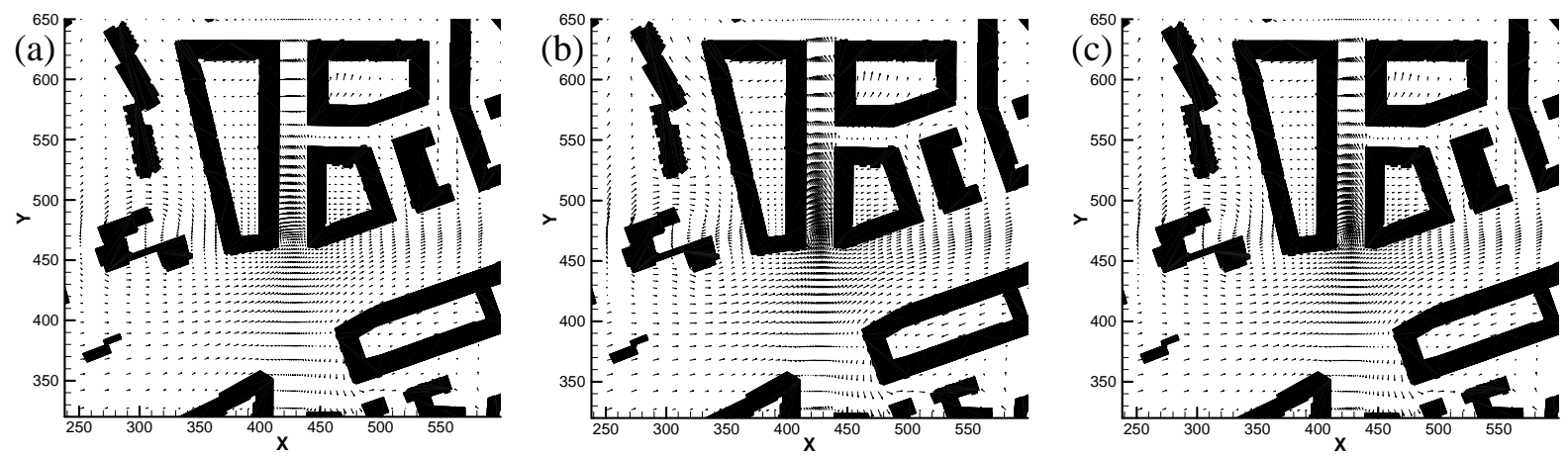

Figure 4. Wind-velocity field at $z=4 m$ for cases: (a) 1 ; (b) 2 ; (c) 3

Figure 5 shows the wind velocity at a lateral plane parallel to the $x$-axis, at $y=500$ and within the street canyon of Runeberg Street, the two lanes of which are also shown. The streetcanyon vortex is evident for all cases and this can explain the upwind accumulation of the concentration within the canyon observed in Fig. 3. As the freestream wind deviates from the westerly direction $\left(270^{\circ}\right)$ and increases in terms of velocity (gradually case 1 then case 3 and finally case 2 ) the centre of the vortex is moved upwards and downwind and the wind velocity within the canyon increases.
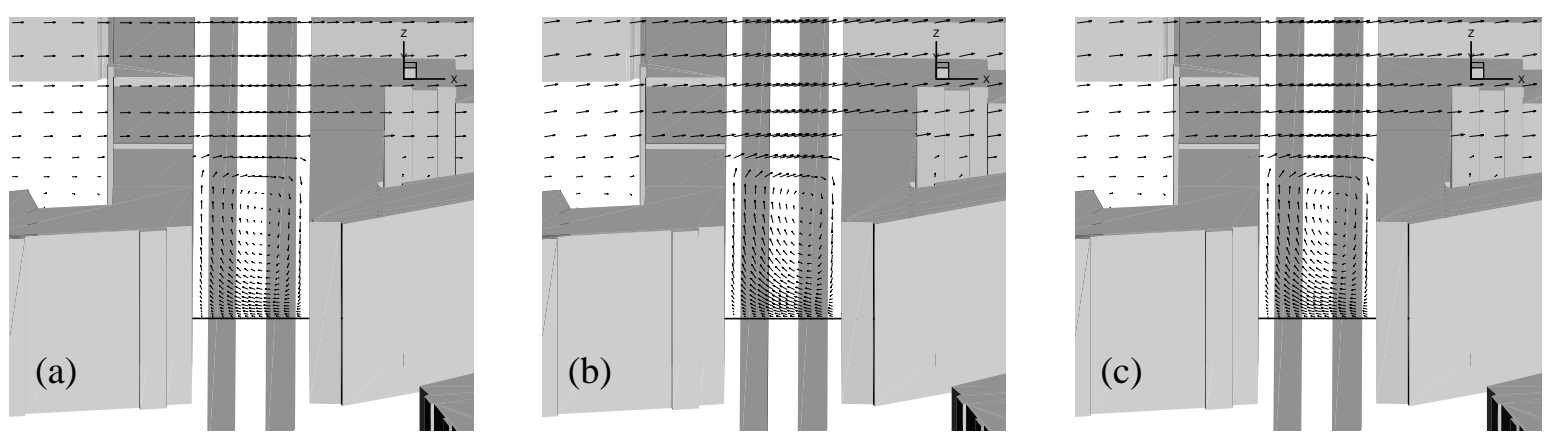

Figure 5 . Wind-velocity at $\mathrm{y}=500 \mathrm{~m}$ for cases: (a) 1 ; (b) 2 ; (c) 3

It is worthwhile pointing out that although velocities within the canyon are higher than at the crossing of Runeberg Street with Hesperian Street (Fig. 3), the street-canyon vortex is responsible for maintaining higher in-canyon concentrations than at the crossing where the concentration is much lower despite of the fact that the emissions at both points are the same. Therefore higher velocities do not necessarily mean higher dispersion rates.

\section{CONCLUSIONS}

Pollution-dispersion modelling in an urban area was carried out using ADREA-HF code. The concentration field within the main street canyon of Runeberg Street is affected by the presence of Hesperian Street that crosses Runeberg Street and more precisely by the wind entering Runeberg Street from Hesperian Street. Furthermore, the fact that Hesperian Street is an open area results in emissions from Hesperian Street barely playing any role to the 
concentration within the street canyon. Finally, deviation of wind from a direction perpendicular to the street canyon and simultaneous increasing of velocity speed causes the in-canyon vortex-centre to move upwards and downwind.

\section{ACKNOWLEDGEMENTS}

The financial support from the European Union under the contract EVK4-CT-2002-00083 is gratefully acknowledged.

\section{REFERENCES}

1. Bartzis J.G. (1991) ADREA-HF: A three-dimensional finite volume code for vapor cloud dispersion in complex terrain, EUR report $13580 \mathrm{EN}$.

2. Kukkonen J., Valkonen E., Walden J., Koskentalo T., Aarnio P., Karppinen A., Bercowicz R., Kartastenpää R. (2001) A measurement campaign in a street canyon in Helsinki and comparison of results with predictions of the OSPM model, Atmos. Environ., 35, 231-243.

3. Kukkonen J., Partanen L., Karppinen A., Walden J., Kartastenpää R., Aarnio P., Koskentalo T., Bercowicz R. (2003) Evaluation of the OSPM model combined with an urban background model against the data measured in 1997 in Runeberg street, Helsinki, Atmos. Environ., 37, 1101-1112.

4. Neofytou P., Venetsanos A.G., Rafailidis S., Bartzis J.G. (2006) Numerical Investigation of the Pollution Dispersion in an Urban Street-Canyon, Environ. Modell. Softw., 21, 525-531.

5. Neofytou P., Venetsanos A.G., Vlachogiannis D., Bartzis J.G, Scaperdas A. (2006) CFD Simulations of the Wind Environment around an Airport Terminal Building, Environ. Modell. Softw., 21, 520-524.

6. Vardoulakis S., Fisher B.E.A., Pericleous K., Gonzalez-Flesca N. (2003) Modelling air quality in street canyons: a review, Atmos. Environ., 37, 155-182.

7. Venetsanos A.G., Bartzis J.G., Würtz J., Papailiou D.D. (2000) Comparative modeling of a passive release from an L-shaped building using one, two and three-dimensional dispersion models, Int. J. Environ. Pollut., 14, 324-333.

8. Walton A., Cheng A.Y.S., Yeung W.C. (2002) Large-eddy simulation of pollution dispersion in an urban street canyon-Part I: comparison with field data, Atmos. Environ., 36, 3601-3613.

9. YTV (2003) Pääkaupunkiseudun liikenne-ennustemallit 2000 (Transport models in the Helsinki metropolitan area 2000). Helsinki Metropolitan Area Council (YTV), Report B 2003:9, pp. 192 (in Finnish). 